\title{
Application of protein profiling of virulent Haemophilus parasuis by MALDI- TOF mass spectrometry
}

\author{
Luisa Z Moreno", Givago FR Silva1', Vasco TM Gomes ${ }^{1}$, Carlos EC Matajira ${ }^{1}$, Ana Paula S Silva ${ }^{1}$, Renan \\ E Mesquita ${ }^{1}$, Nicholas P Lotto ${ }^{1}$, Thais SP Ferreira ${ }^{1}$, Ana Paula G Christ $^{2}$, Maria Inês Z Sato ${ }^{2}$, Yuri \\ Gherpelli ${ }^{3}$, Michele Dottori ${ }^{3}$, Paolo Bonilauri ${ }^{3}$, Andrea Luppi ${ }^{3}$, Andrea M Moreno ${ }^{1}$ \\ ${ }^{1}$ Faculdade de Medicina Veterinária e Zootecnia, Universidade de São Paulo, São Paulo, SP, Brasil \\ ${ }^{2}$ Companhia de Tecnologia de Saneamento Ambiental (CETESB). São Paulo, SP, Brasil \\ ${ }^{3}$ Istituto Zooprofilattico Sperimentale della Lombardia e dell'Emilia Romagna (IZSLER). Brescia, Italia
}

Key words: Haemophilus parasuis, MALDI-TOF MS, serovar, swine.

J Infect Dev Ctries 2016; 10(6):678-681. doi:10.3855/jidc.7787

(Received 09 October 2015 - Accepted 26 November 2015)

Copyright $($ C 2016 Moreno et al. This is an open-access article distributed under the Creative Commons Attribution License, which permits unrestricted use, distribution, and reproduction in any medium, provided the original work is properly cited.

Dear Editor,

Haemophilus parasuis is the causative agent of Glasser's disease and is one of the major opportunistic pathogens in swine intensive production systems, leading to substantial financial losses worldwide [1]. To date, $15 \mathrm{H}$. parasuis serovars have been described varying in virulence and geographical prevalence. However, the existence of a large number of nontypable isolates represents an important challenge for Glasser's disease control, as vaccine immunity presents limited cross-serovar protection [2].

$H$. parasuis is classified as a fastidious organism that is nicotinamide adenine dinucleotide (NAD) dependent, and the standard methods of isolation and biochemical and immunological characterization for this bacterium are considered troublesome and time consuming [3]. Although molecular techniques have succeeded in diagnosing $H$. parasuis [3], the challenges of achieving proper molecular identification and serotyping at the lowest possible cost still remain.

In recent years, matrix-assisted laser desorption/ionization time-of-flight mass spectrometry (MALDI-TOF MS) has become an important bioanalytical diagnostic tool for the detection of protein profiles from whole bacterial cells [4]. This rapid and accurate method can be easily applied to identify bacteria at the genus, species and, in some cases, the subspecies levels [5]. Therefore, MALDI-TOF MS represents a promising alternative to the standard phenotypic and molecular techniques carried out in diagnostic laboratories. In the present study, we assessed the use of MALDI-TOF MS for the characterization of Haemophilus parasuis isolated from different Brazilian states between 2009 and 2014.

A total of $98 \mathrm{H}$. parasuis isolates were studied. The samples were isolated from 66 animals in 40 herds from nine different Brazilian states (Mato Grosso, Mato Grosso do Sul, Minas Gerais, Paraná, Rio Grande do Sul, Santa Catarina, Distrito Federal and São Paulo) in Brazil from 2009 to 2014. Serotyping was previously performed by the immunodiffusion test using autoclaved antigens [6] at the Istituto Zooprofilattico Sperimentale della Lombardia e dell'Emilia Romagna (IZSLER, Italy). H. parasuis identification was also confirmed by PCR based on species-specific amplification of the 16S rRNA gene, as described by Oliveira et al. [7]. All isolates were also positive for the amplification of the group $1 \mathrm{vtaA}$ gene [8] that has been associated with virulent $H$. parasuis strains.

For MALDI-TOF MS sample preparation, bacterial proteins were extracted using an ethanol/formic acid protocol [9]. The protein suspension $(1 \mu \mathrm{l})$ was transferred to a polished steel MALDI target plate (Bruker Daltonik, Bremen, Germany) and allowed to dry at room temperature. The sample was overlaid with $1 \mu 1$ of matrix (10 mg $\alpha$-cyano-4-hydroxy-cinnamic acid $\mathrm{ml}^{-1}$ in $50 \%$ acetonitrile/ $2.5 \%$ trifluoroacetic acid), and mass spectra in the 2-20 kDa range were acquired using a Microflex mass spectrometer (Bruker Daltonik). 
Figure 1: Dendrogram showing the relationship among the $H$. parasuis isolates spectral profiles.

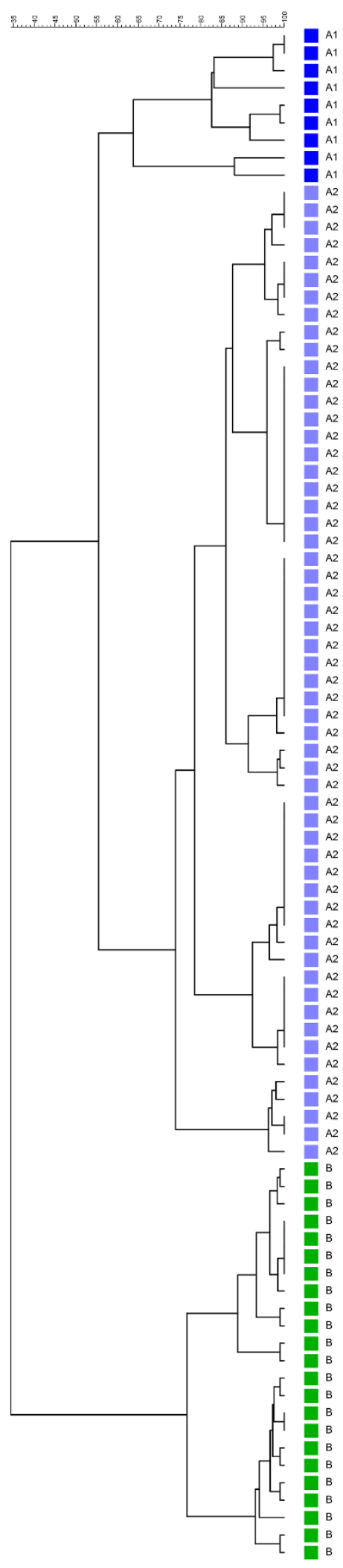

\begin{tabular}{|c|c|c|c|c|c|c|}
\hline Isolate & Animal & Herd & Organ & Serotype & State & Year \\
\hline Hp113 & 64 & G40 & Lung & NT & PR & 2013 \\
\hline Hp114 & 64 & G40 & Lung & NT & PR & 2013 \\
\hline Hp94 & 54 & G36 & Lung & NT & $\mathrm{sc}$ & 2012 \\
\hline Hр89 & 50 & G33 & Lung & NT & SP & 2012 \\
\hline Hp122 & 69 & G44 & Lung & NT & SP & 2013 \\
\hline Hp123 & 69 & G44 & Lung & NT & SP & 2013 \\
\hline Hp121 & 68 & G43 & Lung & NT & RS & 2013 \\
\hline Hp108 & 61 & G38 & Lung & 4 & SP & 2012 \\
\hline Hр32 & 19 & G16 & Lung & NT & MS & 2010 \\
\hline Hp145 & 86 & G55 & Lung & 4 & MT & 2014 \\
\hline Hp148 & 88 & G55 & Lung & 4 & MT & 2014 \\
\hline Hp92 & 52 & G36 & Lung & NT & sc & 2012 \\
\hline Hp11 & 8 & G8 & Lung & 14 & MT & 2010 \\
\hline Hp133 & 77 & G49 & Lung & NT & SP & 2013 \\
\hline Hр24 & 15 & G12 & Lung & 4 & PR & 2010 \\
\hline Hp75 & 42 & G28 & Lung & NT & MT & 2011 \\
\hline Hp18 & 12 & G12 & Lung & 4 & PR & 2010 \\
\hline Hp111 & 63 & G39 & Lung & 4 & SP & 2013 \\
\hline Hp139 & 82 & G52 & Lung & 4 & PR & 2014 \\
\hline Hp104 & 59 & G38 & Lung & 4 & SP & 2012 \\
\hline Hp127 & 72 & G46 & Lung & NT & MG & 2013 \\
\hline Hp129 & 73 & G46 & Lung & NT & MG & 2013 \\
\hline Hp144 & 86 & G55 & Lung & 4 & MT & 2014 \\
\hline Hp146 & 86 & G55 & Lung & 4 & MT & 2014 \\
\hline Hp15 & 10 & G10 & Lung & NT & MG & 2010 \\
\hline Hр21 & 13 & G12 & Lung & NT & PR & 2010 \\
\hline Hp23 & 14 & G12 & pericardium & NT & PR & 2010 \\
\hline Hр27 & 16 & G13 & Lung & 14 & PR & 2010 \\
\hline Hp65 & 36 & G25 & Lung & NT & sc & 2011 \\
\hline Hp97 & 56 & G36 & Lung & NT & $\mathrm{sc}$ & 2012 \\
\hline Hp105 & 60 & G38 & Lung & NT & SP & 2012 \\
\hline Hp116 & 65 & G41 & Lung & 4 & PR & 2013 \\
\hline Hp13 & 9 & G9 & Lung & NT & MT & 2010 \\
\hline Hp19 & 12 & G12 & Lung & 4 & PR & 2010 \\
\hline Hp4 & 3 & G2 & Lung & NT & MS & 2009 \\
\hline Hp43 & 25 & G22 & Lung & 4 & sc & 2011 \\
\hline Hp6 & 5 & G5 & Lung & NT & SP & 2010 \\
\hline Hp7 & 5 & G5 & Lung & NT & SP & 2010 \\
\hline Hр79 & 44 & G30 & Lung & 14 & RS & 2011 \\
\hline Hp9 & 6 & G6 & pericardium & NT & $P R$ & 2010 \\
\hline Hр26 & 16 & G13 & Lung & 14 & PR & 2010 \\
\hline Hp28 & 17 & G14 & Lung & 4 & PR & 2010 \\
\hline Hр29 & 17 & G14 & Lung & 4 & PR & 2010 \\
\hline Hp54 & 30 & G24 & Lung & 5 & MG & 2011 \\
\hline Hp12 & 8 & G8 & Lung & 14 & MT & 2010 \\
\hline Hp140 & 83 & G53 & Lung & 4 & SP & 2014 \\
\hline Hp25 & 15 & G12 & Lung & 4 & PR & 2010 \\
\hline Hр3 & 2 & G1 & Lung & 13 & SP & 2009 \\
\hline Hp80 & 45 & G30 & Lung & 14 & RS & 2011 \\
\hline Hp81 & 46 & G30 & Lung & 14 & RS & 2011 \\
\hline Hp88 & 49 & G32 & Lung & 1 & PR & 2012 \\
\hline Hp93 & 53 & G37 & Lung & 13 & $\mathrm{sc}$ & 2012 \\
\hline Hp22 & 14 & G12 & pericardium & NT & PR & 2010 \\
\hline Hp141 & 83 & G53 & Lung & 4 & SP & 2014 \\
\hline Hp101 & 58 & G37 & Lung & NT & sc & 2012 \\
\hline Hp106 & 60 & G38 & Lung & 4 & SP & 2012 \\
\hline Hp147 & 87 & G55 & Lung & 4 & MT & 2014 \\
\hline Hp2O & 13 & G12 & Lung & NT & PR & 2010 \\
\hline Hp98 & 56 & G36 & Lung & NT & sc & 2012 \\
\hline Hp128 & 72 & G46 & Lung & NT & MG & 2013 \\
\hline Hp103 & 59 & G38 & Lung & 4 & SP & 2012 \\
\hline Hp138 & 81 & G51 & Lung & 5 & PR & 2013 \\
\hline Hp124 & 70 & G45 & Lung & 5 & PR & 2013 \\
\hline Hp125 & 70 & G45 & Lung & 5 & PR & 2013 \\
\hline Hp120 & 67 & G42 & Lung & NT & MG & 2013 \\
\hline Hp45 & 26 & G23 & pericardium & NT & sc & 2011 \\
\hline Hp64 & 36 & G25 & Lung & NT & sc & 2011 \\
\hline Hp61 & 34 & G25 & Lung & NT & sc & 2011 \\
\hline Hр35 & 20 & G17 & Lung & 5 & PR & 2010 \\
\hline Hp46 & 26 & G23 & pericardium & NT & sc & 2011 \\
\hline Hp8 & 6 & G6 & pericardium & NT & PR & 2010 \\
\hline Hp85 & 28 & G23 & Lung & NT & sc & 2011 \\
\hline Hр34 & 20 & G17 & Lung & 4 & PR & 2010 \\
\hline Hp57 & 32 & G25 & Lung & 13 & sc & 2011 \\
\hline Hp58 & 32 & G25 & Lung & 13 & sc & 2011 \\
\hline Hp83 & 47 & G31 & Lung & NT & MT & 2011 \\
\hline Hp84 & 47 & G31 & Lung & NT & MT & 2011 \\
\hline Hp126 & 71 & G45 & Lung & 5 & PR & 2013 \\
\hline Hp72 & 40 & G27 & Lung & 4 & DF & 2011 \\
\hline Hp66 & 37 & G25 & thoracic cavity & 5 & sc & 2011 \\
\hline Hp76 & 43 & G29 & Lung & NT & RS & 2011 \\
\hline Hp119 & 67 & G42 & Lung & 13 & MG & 2013 \\
\hline Hp67 & 37 & G25 & thoracic cavity & 5 & sc & 2011 \\
\hline Hp107 & 61 & G38 & Lung & 4 & SP & 2012 \\
\hline Hp118 & 66 & G42 & Lung & 13 & MG & 2013 \\
\hline Hp137 & 80 & G51 & Lung & 5 & PR & 2013 \\
\hline Hp2 & 2 & G1 & Lung & 13 & SP & 2009 \\
\hline Hp70 & 39 & G27 & Lung & 4 & DF & 2011 \\
\hline
\end{tabular}


Each sample was distributed over six spots and measured four times, resulting in a total of 24 spectra per isolate.

For the MALDI-TOF identification, the spectra were loaded into MALDI BioTyper 3.0 and compared with the manufacturer's library, which resulted in the $\log$ (score) value. Standard Bruker interpretative criteria were applied; scores $\geq 2.0$ were accepted for species assignment and scores $\geq 1.7$ but $\leq 2.0$ for genus identification. For further analysis, at least 20 technical spectral replicates were used to generate a main spectrum for each isolate in Bionumerics 7.5 (Applied Maths NV, Saint-Martens-Latem, Belgium). Cluster analysis was performed using the number of different peaks detected and UPGMA method; principal component analysis (PCA) was applied to analyze the homogeneity of the $H$. parasuis spectra.

All isolates were identified using MALDI BioTyper with $\log$ (score) values $>2.0$ for $H$. parasuis; a mean $\log$ (score) value of 2.226 was calculated. Furthermore, a slight variation among replicates for each isolate was observed, which corroborates the importance of using more than one spot per sample and even more than one spot measure to ensure bacterial identification and its reproducibility. Although MALDI-TOF MS has been validated for the identification of several microorganisms $[5,10]$, the technique still presents some limitations with regard to spectral variation and reproducibility. The main causes for this are bacterial growth conditions and protein extraction methods [10].

To avoid interference of the medium used for bacterial isolation and maintenance, the culture broth was initially centrifuged; the pellet was washed with TE $(\mathrm{pH}$ 8.0) and then dissolved in deionized water for further protein extraction. In the initial MALDI-TOF MS identification studies, the protein purification or extraction step was only applied when no identification was obtained using the direct colony transfer method. As it has been proven that protein extraction improves identification yield, this step has been integrated into MALDI protocols, especially for intra-species and strain analyses $[11,12]$.

The feasibility of using MALDI-TOF MS for $H$. parasuis identification was expected, as Kuhnert et al. [9] previously described the MALDI-TOF MS application for Pasteurellaceae identification from animals. However, the comparison of the protein profiles by clustering and PCA has not been reported yet. The protein spectral cluster analysis separated the H. parasuis isolates into two main groups, A and B (Figure 1), and the group A further divides into two subgroups (A1 and A2). The PCA also confirmed this
Figure 2: Principle Component Analysis (PCA) of the $H$. parasuis protein spectral profiles. Isolates coloring corresponds to respective serovars (NT - non typeable).

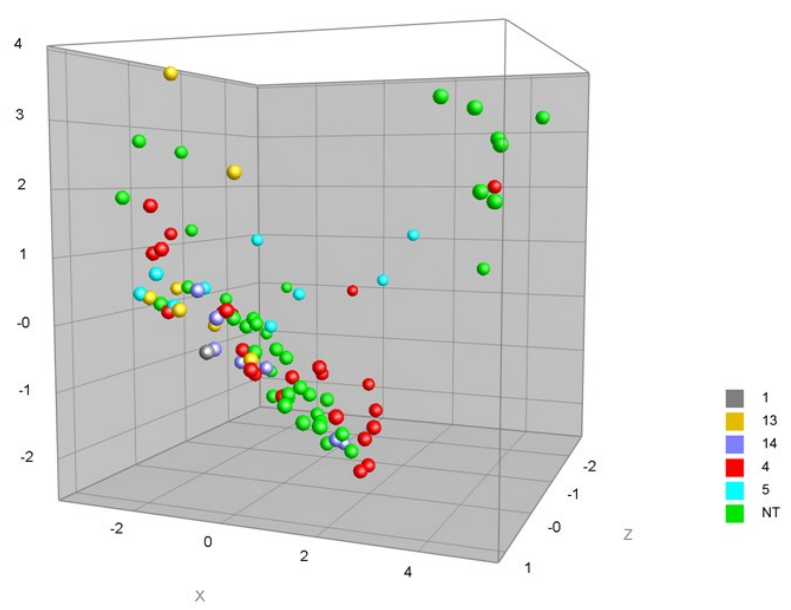

clustering tendency (Figure 2). Although it was possible to observe intra-group similarity among the $H$. parasuis isolates, no relationship between the spectral clusters and the isolate's origin and serovar was observed.

As only abundant housekeeping proteins, particularly ribosomal proteins, are detected in MALDI-TOF MS analysis [13] and the ethanol/formic acid protocol results in total bacterial protein extraction, this could explain the lack of association among the spectral clusters and the isolate's origin and intrinsic characteristics. Moreover, the intra-species variation demonstrated by the spectral cluster analysis has also been found in $H$. parasuis molecular typing $[14,15]$. As the MALDI-TOF MS technique is improving for specific protein detection, it could be used for further $H$. parasuis applied research particularly for characterizing membrane proteins patterns among serovars due to its association with virulence.

\section{Acknowledgements}

This study was supported by FAPESP (grant 2012/19154-5), CAPES and CNPq.

\section{References}

1. Xu Z, Yue M, Zhou R, Jin Q, Fan Y, Bei W, Chen H (2011) Genomic Characterization of Haemophilus parasuis SH0165, a Highly Virulent Strain of Serovar 5 Prevalent in China. PLoS ONE 6: e19631.

2. Bak H, Riising HJ (2002) Protection of vaccinated pigs against experimental infections with homologous and heterologous Haemophilus parasuis. Vet Res 151: 502-505.

3. Howell KJ, Weinert LA, Chaudhuri RR, Luan SL, Peters SE, Corander J, Harris D, Angen $\varnothing$, Aragon V, Bensaid A, Williamson SM, Parkhill J, Langford PR, Rycroft AN, Wren BW, Holden MTG, Tucker AW, Maskell DJ (2014) The use of genome wide association methods to investigate pathogenicity, 
population structure and serovar in Haemophilus parasuis. BMC Genomics 15:1179.

4. Carbonnelle E, Mesquita C, Bille E, Day N, Dauphin B, Beretti JL, Ferroni A, Gutmann L, Nassif X (2011) MALDI-TOF mass spectrometry tools for bacterial identification in clinical microbiology laboratory. Clin Biochem 44: 104-109.

5. Kliem M, Sauer S (2012) The essence on mass spectrometry based microbial diagnostics. Curr Opin Microbiol 15: 397-402.

6. Kielstein P, Rapp-Gabrielson VJ (1992) Designation of 15 serovars of Haemophilus parasuis on the basis of immunodiffusion using heat-sable antigen extracts. J Clin Microbiol 30: 862-865.

7. Oliveira S, Galina L, Pijoan C (2001) Development of a PCR test to diagnose Haemophilus parasuis infections. J Vet Diagn Invest 13: 495-501.

8. Olvera A, Pina S, Macedo N, Oliveira S, Aragon V, Bensaid A (2012) Identification of potentially virulent strains of Haemophilus parasuis using a multiplex PCR for virulence associated autotransporters (vtaA). Vet J 191: 213-218.

9. Kuhnert P, Bisgard M, Korczak BM, Schwender S, Christensen H, Frey J (2012) Identification of animal Pasteurellacea by MALDI-TOF mass spectrometry. J Microbiol Methods 89: 1-7.

10. Biswas S, Rolain JM (2013) Use of MALDI-TOF mass spectrometry for identification of bacteria that are difficult to culture. J Microbiol Methods 92: 14-24.

11. Alatoom AA, Cunningham SA, Ihde SM, Mandrekar J, Patel R (2011) Comparison of direct colony method versus extraction method for identification of Gram-positive cocci by use of Bruker Biotyper matrix-assisted laser desorption ionization- time of flight mass spectrometry. J Clin Microbiol 49: 28682873.

12. Fournier R, Wallet F, Grandbastien B, Dubreuil L, Courcol R, Neut C, Dessein R (2012) Chemical extraction versus direct smear for MALDI-TOF mass spectrometry identification of anaerobic bacteria. Anaerobe 18: 294-297.

13. Sauer S, Kliem M (2010) Mass spectrometry tools for the classification and identification of bacteria. Nat Rev Microbiol 8: 74-82.

14. Mullins MA, Register KB, Brunelle BW, Aragon V, GalofreMila N, Bayles DO, Jolley KA (2013) A curated public database for multilocus sequence typing (MLST) and analysis of Haemophilus parasuis based on an optimized typing scheme. Vet Microbiol 162: 899-906.

15. Olvera A, Segalés J, Aragón V (2007) Update on the diagnosis of Haemophilus parasuis infection in pigs and novel genotyping methods. Vet J 174: 522-529.

\section{Corresponding author}

Andrea M. Moreno. FMVZ/USP

Av Prof Dr Orlando Marques de Paiva, 87, Cidade Universitária, CEP 05508 270. São Paulo/ SP - Brazil.

Phone + $55021113091-1377$

Fax: +55 $021113091-7928$

Email: morenoam@usp.br

Conflict of interests: No conflict of interests is declared. 